\title{
BALLNESS É TÃO EFICAZ QUANTO IOGA NA PROMOÇÃO DE FLEXIBILIDADE EM MULHERES DE MEIA-IDADE
}

\author{
Laisa Cruz Torres Vieira ${ }^{1}$ \\ Jefferson Fernando Coelho Rodrigues Júnior ${ }^{2}$ \\ Neivan José Andrade Magalhães ${ }^{3}$ \\ Caio Victor Coutinho de Oliveira ${ }^{4}$ \\ Sergio Luiz Galan Ribeiro 5 \\ Marcos Antonio Pereira dos Santos ${ }^{6}$
}

\begin{abstract}
RESUMO
Introdução: A incorporação da prática de modalidades alternativas de exercício para promoção da flexibilidade é cada vez mais comum. Estudos demonstram associação entre índices de flexibilidade e qualidade de vida. Nesse contexto, a ioga e o ballness surgem como ferramentas capazes de aumentar a flexibilidade dos indivíduos. Objetivo: Comparar os níveis de flexibilidade da flexão do tronco de mulheres de meia-idade praticantes de ballness e de ioga. Métodos: 24 mulheres de meia-idade foram voluntariamente selecionadas. Foram divididas em três grupos ( $\mathrm{n}=8 / \mathrm{grupo})$ : controle (C) (48,87 $\pm 2,80$ anos), ballness (B) (48,62 $\pm 5,63 a n o s)$ e ioga (I) (49,12 $\pm 5,48$ anos). Para inclusão no estudo os sujeitos de (B)e (I) deveriam ser praticantes há seis meses da modalidade, com frequência mínima de duas sessões/semana. Ademais, toda amostra deveria estar isenta de lesão na região lombo sacra, no quadril e na musculatura dos membros inferiores. A flexibilidade foi mensurada por meio do banco de Wells, utilizando o post hoc de Tukey para comparação entre tratamentos ( $\mathrm{p}<0,05)$. Resultados: A flexibilidade dos grupos exercícios (B e I) revelou-se maior que

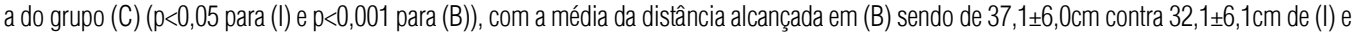
$20,5 \pm 3,1 \mathrm{~cm}$ de (C), sem diferenças entre (B) e (I). Adicionalmente, no teste de "sentar e alcançar", os sujeitos dos grupos (B) e (I) apresentaram prevalência do nível "bom" de 37\% e 37,5\%, respectivamente, enquanto que ninguém de (C) mostrou-se nessa condição. Conclusões: A prática regular de ballness e de ioga em mulheres de meia-idade são suficientes para promover maior flexibilidade em comparação às sedentárias.
\end{abstract}

Palavras-chave: Exercício. Flexibilidade. Sedentarismo.

\begin{abstract}
Introduction: The practice's incorporation of the exercises modalities alternatives for flexibility promotion is more and more common. Studies demonstrate association between flexibility and life quality. In this context, yoga and ballness arise as tools capable of improving the individual's flexibility. Objective: To compare the flexibility levels of the trunk flexion of the middle-age women of the ballness practioners and yoga. Metho-

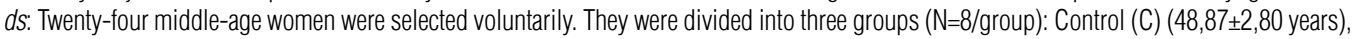
Ballness (B) $(48,62 \pm 5,63$ years) and yoga (I) $(49,12 \pm 5,48$ years), for inclusion in the study, the subjects of (B) and (I) should be practicing the modality for six months, with a minimum frequency of two meetings a week. Besides, the entire sample should be free from injuries in the lumbosacral region, in the hip and in the lower members of the musculature. Results: The flexibility of the exercises groups proved to be better than that of $(C)$ group $(p<0,05$ for (I) $p<0,001$ in (B), the average reached distance of (B) was $37,1 \pm 6,0 \mathrm{~cm}$ against $32,1 \pm 6,1 \mathrm{~cm}$ of $(\mathrm{I})$ and of $20,5 \pm 3,1 \mathrm{~cm}(\mathrm{C})$, no differences between (B) an (I). Additionally, in the 'sit and reach' test, the subject of the groups (B) and (I) had a "good" prevalence level of $37 \%$ and $37,5 \%$ respectively, whereas nobody of (C) showed in this condition. Conclusions: The regular practice of the ballness yoga and middle-age women, even with low weekly frequency, are sufficient to promove greater flexibility compared to sedentary.
\end{abstract}

Keywords: Exercise. Flexibility. Sedentary lifestyle.

\footnotetext{
1 Graduado em Educação Física pela Universidade Estadual do Piauí. laisa-cruz@hotmail.com

2 Acadêmico do Curso de Educação Física. Universidade Federal do Piauí. jefferssonfernando@hotmail.com

3 Graduado em Educação Física pela Universidade Estadual do Piauí. neivanjose02@hotmail.com

${ }^{4}$ Bacharel em Ciências da Nutrição pela Universidade Federal da Paraíba, mestre pelo Programa de Pós-Graduação em Ciências da Nutrição (UFPB). caiovco@gmail.com

5 Graduado em Educação Física pela Escola Superior de Educação Física e Desportos de Catanduva. Mestrado em Master of Science - George Peabody College For Teacher - Nashville Tn USA e Doutorado em Doctor of Education - University Of Georgia USA. sergiogalan7@hotmail.com

${ }^{6}$ Graduado em Educação Física pela Universidade Estadual do Piauí. Doutor na Área de Biotecnologia-Renorbio marcosedfisio@gmail.com
} 
O envelhecimento é fenômeno natural que envolve fatores biológicos, ambientais e psicológicos (Gonçalves et al., 2011). É associado com modificações em diversos sistemas fisiológicos, podendo impactar na capacidade de realização das atividades diárias (Donnelly et al., 2009), autonomia e independência (Krinski et al., 2008; Lojudice et al., 2008).

Mudanças na função cardíaca, pressão sanguínea, função vascular, composição corporal, força muscular, controle motor e flexibilidade são comuns no envelhecimento (SINGH, 2004). Por outro lado, exercícios físicos são capazes de atenuar o processo do envelhecimento ou reverter perdas de capacidades físicas como força, equilíbrio e flexibilidade, principalmente em atividades da vida diária (Donnelly et al., 2009).

Já é bem demonstrada a relação benéfica entre prática de exercício e envelhecimento saudável apontando benefícios de longevidade à população que pratica exercícios desde jovem (Matsudo; Matsudo; Barros Neto, 2001; Westerterp, 2000). Exercícios de leve a moderada intensidade, como em atividades aeróbias, são preferidos pelos idosos (Rafferty et al., 2002). O interesse por formas alternativas de exercício para promoção de seus benefícios tem sido crescente (Sherman et al., 2010; Macy, 2008). Nesse contexto, a ioga, prática indiana milenar, vem sendo crescentemente adotada como prática promotora de saúde no Ocidente, mostrando-se eficaz para melhorar, entre outros aspectos, a flexibilidade (Raub, 2002).

Ioga corresponde a um grupo de exercícios que trabalham de forma harmônica o corpo e a mente, com auxílio de técnicas posturais e de respiração (De Barros et al., 2014). A prática de ioga gera uma disciplina espiritual e emocional, além de equilíbrio, coordenação, força e flexibilidade (Raub, 2002). Esta modalidade também se mostra eficaz no tratamento de doenças, como dores de coluna (Williams, 2005) e cardiopatias (Pullen et al., 2010) e em parâmetros de saúde como adaptações cardiorrespiratórias (Balasubramanian; Pansare, 1991; Baptista; Dos Santos Alves, 2005), composição corporal (Chen et al., 2008) e estado de equilíbrio (Gauchard et al., 1999). Há grande variedade nas modalidades de ioga, podendo-se citar Iyengar (Dibenedetto et al., 2005), Silver Ioga (Chen et al., 2007) e hatha, a mais estudada (Clay et al., 2005).

Nesse contexto, é importante elucidar se formas alternativas de exercício, nesse caso o ballness, são realmente eficazes para uma melhora da aptidão física e flexibilidade (Macy, 2008). O ballness é uma nova modalidade de exercício que compreende um conjunto de técnicas de exercícios desenvolvidos a partir do uso terapêutico da bola suíça. Propõe-se desenvolver capacidades físicas e habilidades motoras como flexibilidade, força, coordenação, equilíbrio, resistência e consciência corporal, melhorando o controle motor, a percepção corporal e consciência do movimento (Gonçalves et al., 2011; Bijlani; Vempati; Yadav, 2005; Sharma; Gupta; Bijlani, 2008). Tanto o ballness quanto a ioga trabalham a flexibilidade do tronco, caracterizada por se utilizar da amplitude de movimento sem ênfase na velocidade, com manutenção de determinada posição de uma articulação sobre um período de tempo (Williams et al., 2005).

Apesar da similaridade, enquanto a ioga é uma pratica milenar, o ballness é modalidade recente, que surge como uma forma alternativa de trabalhar o corpo como um todo (Okumura; Silva, 2009). Por outro lado, se os efeitos benéficos da ioga também são obtidos pelo ballness é questão que ainda não foi elucidada na literatura prévia. De fato, estudos acerca do ballness e suas propriedades terapêuticas na capacidade funcional são inexistentes. Assim, supomos que a prática regular de ballness será capaz de promover respostas similares às da ioga em mulheres de meia-idade.

Desta forma, o presente estudo teve por objetivo comparar os níveis de flexibilidade do tronco de mulheres de meia-idade praticantes das modalidades ballness e ioga.

\section{MÉTODOS}

\section{Desenho experimental}

O estudo foi conduzido com mulheres matriculadas nas modalidades ballness e ioga em um clube social do município de Teresina - PI, Brasil. 
Para tanto, selecionou-se 24 mulheres para composição da amostra, as quais foram divididas em três grupos (ballness, ioga e controle). Estas foram acompanhadas durante seis meses, sendo avaliadas antes e ao final do protocolo experimental. A todas as voluntárias foi explicado previamente sobre todo o projeto, seus objetivos, metas e a metodologia a ser utilizada, deixando claro que poderiam ou não aceitar participar de forma voluntária da pesquisa.

\section{Sujeitos do estudo}

A amostra, composta apenas por mulheres $(48,5 \pm 4,7$ anos), foi dividida em três grupos $(\mathrm{n}=8)$ : grupo ballness $(B)\left(23,78 \pm 0,9 \mathrm{Kg} \cdot \mathrm{m}^{-2}\right)$, grupo ioga (I) $\left(23,36 \pm 4,3 \mathrm{Kg} . \mathrm{m}^{-2}\right)$ e controle (C) $\left(27,57 \pm 2,4 \mathrm{Kg} \cdot \mathrm{m}^{-2}\right)$. As participantes dos grupos (B) e (I) deveriam ser praticantes há mais de seis meses da modalidade. Toda a amostra deveria estar isenta de lesão na região lombo-sacra, no quadril e na musculatura dos membros inferiores, como forma de evitar alteração nas mensurações de flexibilidade. Todas assinaram o Termo de Consentimento Livre e Esclarecido. A pesquisa foi desenvolvida em concordância com as normas vigentes envolvendo seres humanos segundo princípios éticos estabelecidos na Resolução $N^{\circ}$ 466, de 12 de dezembro de 2012 pelo Conselho Nacional de Saúde. O projeto foi submetido ao Comitê de Ética do Instituto Camillo Filho e aprovado conforme número 1942.0.000.044-10. Os dados de caracterização dos grupos estão apresentados na Tabela 1.

\section{Instrumentos da coleta de dados}

Para o calculo do IMC das voluntárias foram coletados o peso e a estatura, e posteriormente os dados foram classificados segundo a Sociedade Brasileira de Endocrinologia e Metabologia. Para avaliação da flexibilidade da articulação do tronco foi utilizado o banco de Wells, que consiste em uma caixa de "sentar e alcançar" (Banco de Wells Instant Flex Sanny BW2002). A caixa possui dimensões de $30,5 \mathrm{~cm} \times 30,5 \mathrm{~cm} \times 50,6 \mathrm{~cm}$, com uma régua em centímetros e uma marcação com deslizamento específico. Os resultados da flexibilidade do tronco foram avaliados de acordo com a classificação da tabela de Sentar e Alcançar proposto por Wells e Dillon (1952), que possui cinco classificações: fraco, regular, médio, bom e ótimo.

\section{Procedimentos da coleta de dados}

Os testes foram realizados antes do exercício, sempre entre $17 \mathrm{~h} 30 \mathrm{~min}$ e $18 \mathrm{~h} 30 \mathrm{~min}$ Neste momento o avaliado assume a posição sentada, com os pés apoiados na plataforma do banco de Wells. $\mathrm{O}$ avaliador então apoia os joelhos do avaliado, evitando que estes se flexionem; a seguir o avaliado flexiona o tronco vagarosamente à frente o máximo que puder. Os voluntários realizaram três tentativas seguidas, sendo considerada na análise dos dados a média das três tentativas.

\section{Protocolo de treinamento}

O grupo controle, assim como os demais grupos, participou de todas as avaliações, no mesmo período, ficando isento apenas do protocolo de treinamento. Analisando as duas modalidades no que se refere aos procedimentos adotados para desenvolver a flexibilidade, observa-se que ambas utilizam o alongamento passivo e estático, respirando normalmente e pelas narinas, com 50 minutos cada sessão. $\mathrm{O}$ ponto que diferencia as atividades reside no fato de que no ballness, os exercícios de flexibilidade são realizados com a bola suíça de tamanho médio. São executados em média cinco repetições, permanecendo na posição estática entre 10 e 15 segundos, com intervalos de 20 segundos entre uma repetição e outra. Os exercícios de flexibilidade foram executados sem ajuda do professor, respeitando os limites de flexibilidade de cada aluno. Além disso, foram realizados exercícios voltados para o trabalho de força (dinâmica) começando com 3 séries, entre 5 e 8 repetições. O aumento da quantidade de repetições acontecia na medida em que o condicionamento de cada aluna ia evoluindo, aumentando em média 3 repetições por mês. A cada repetição eram 
respeitadas as seguintes fases: inspiração, retenção e expiração, com intervalos de 2 ou 3 respirações entre uma repetição e outra.

\section{Tratamento Estatístico}

Os dados estão apresentados por meio de estatística descritiva de média, desvio padrão da média e porcentagem. Realizou-se o teste de Levene para verificação da validade dos dados. Para comparar medidas de flexibilidade entre os grupos e pelo fato de ser apenas um fator foi utilizada a variância Anova one way seguida pelo post hoc de Tukey. O estudo admitiu o nível de significância com $\mathrm{p}<0,05$. Utilizou-se o software Instat versão 3.0.

\section{RESULTADOS}

A amostra foi composta por mulheres de meia idade, com IMC variando entre eutrofia e sobrepeso. Durante o período experimental, nenhuma delas apresentou reação adversa a quaisquer intervenções. As suas características estão descritas na tabela 1.

Tabela 1 - Caracterização antropométrica da amostra do estudo

\begin{tabular}{|c|c|c|c|}
\hline & $\begin{array}{l}\text { Controle } \\
(\mathrm{n}=8)\end{array}$ & $\begin{array}{l}\text { Ballness } \\
(\mathrm{n}=8)\end{array}$ & $\begin{array}{l}\text { Ioga } \\
(n=8)\end{array}$ \\
\hline Idade (anos) & $48,87 \pm 2,80$ & $48,62 \pm 5,63$ & $49,12 \pm 5,48$ \\
\hline Peso (kg) & $67,37 \pm 7,99$ & $58,6 \pm 3,25$ & $58,75 \pm 7,83$ \\
\hline Altura (m) & $1,56 \pm 0,05$ & $1,57 \pm 0,04$ & $1,59 \pm 0,07$ \\
\hline IMC $\left(\mathrm{kg} / \mathrm{m}^{2}\right)$ & $27,57 \pm 2,13$ & $23,77 \pm 0,89$ & $23,35 \pm 4,30$ \\
\hline
\end{tabular}

Peso em $\mathrm{Kg}$, Estatura em centímetros, IMC em $\mathrm{Kg} / \mathbf{m}^{2}$ e Idade em anos. Os dados estão apresentados como média+desvio padrão da média; não foram encontradas diferenças significativas entre os grupos. Os grupos ioga, ballness e controle não apresentaram diferenças significativas quanto às medidas antropométricas.

Fonte: Dados coletados pelos autores.

Classificação dos grupos no teste de sentar e alcançar: Os grupos (B) e (I) apresentaram maior prevalência nos níveis "bom" e "ótimo" (50\% e 37\% para (B) e $25 \%$ e $37,5 \%$ para (I), respectivamente). O grupo (C), por sua vez, teve prevalência de $75 \%$ e
$25 \%$ nos níveis "fraco" e "regular", respectivamente, não apresentando nenhuma sedentária nos níveis "ótimo", "bom" e "médio" (Figura 1).

Figura 1 - Classificação dos grupos de acordo com a tabela de "Sentar e alcançar"

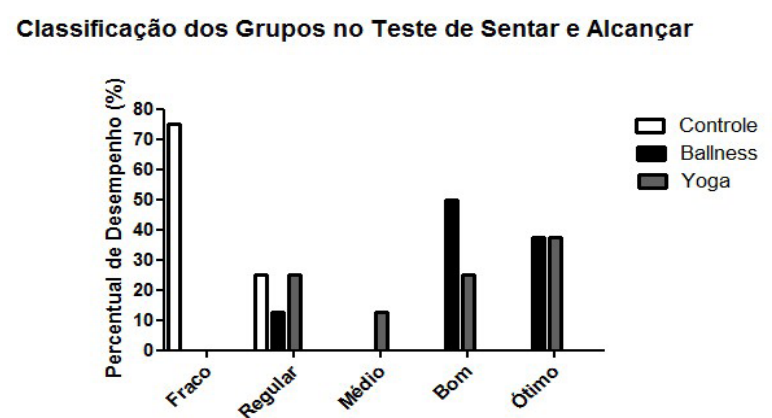

Fonte: Dados coletados pelos autores

\section{Comparação intergrupos na distância alcançada}

A comparação intergrupos é apresentado na Figura 2. A média da distância alcançada do grupo (B) foi de $37,1 \pm 6,0 \mathrm{~cm}$ contra $32,1 \pm 6,1 \mathrm{~cm} \mathrm{e}$ $20,5 \pm 3,1 \mathrm{~cm}$ de (I) e (C), respectivamente. Entre (I) e $(B)$, não houve diferenças estatísticas ( $\mathrm{p}>0.05)$. Por outro lado tanto a flexibilidade das praticantes de (I) $(\mathrm{p}<0,05)$ quanto das praticantes de $(B)$ $(\mathrm{p}<0,001)$ foi maior que as do grupo de $(C)$, como demonstrado pelo teste de tukey.

Figura 2 - Comparação entre os grupos (C), (B) e (I) em relação à distância alcançada $(\mathrm{cm})$

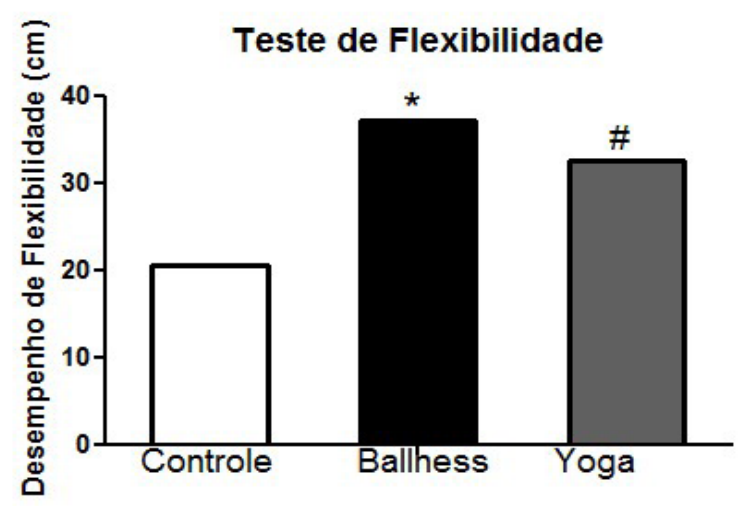

Fonte: Dados coletados pelos autores. 


\section{DISCUSSÃ̃o}

O principal resultado do presente trabalho foi a constatação de que mulheres de meia-idade saudáveis que praticam regularmente o ballness apresentam flexibilidade da articulação do tronco similar àquelas que são praticantes de ioga.

No envelhecimento há diminuição da resistência e mobilidade articular como principais alterações no sistema neuromuscular, repercutindo, assim, na flexibilidade (Telles; Dash; Naveen, 2009). Diversos estudos verificaram os efeitos preventivos/ terapêuticos de diferentes modalidades de ioga na capacidade funcional de indivíduos, estando estes sadios (Bijlani; Vempati; Yadav, 2005) ou doentes (Sharma; Gupta; Bijlani, 2008) (Pearson, 2000). Além disso, como já bem evidencia a literatura, indivíduos praticantes destas modalidades mostram resultados superiores aos sedentários (Clay et al., 2005; Pearson, 2000). Assim, investigar como modalidades alternativas às clássicas, além da ioga, podem afetar a flexibilidade é tema relevante para pesquisas. Neste contexto, até onde sabemos, este é o primeiro estudo que analisou o potencial da modalidade de ballness para melhorar a flexibilidade de mulheres de meia-idade.

Levando em consideração a classificação proposta por Wells e Dillon (1952), as mulheres sedentárias apresentaram resultados menos expressivos do que as praticantes de B e I, apresentando ausência de participantes com níveis "bom" ou "ótimo". Este fato demonstra o efeito do sedentarismo na flexibilidade e conseguinte redução dessa capacidade funcional (Thaash; Kell, 1987).

Interessante notar que os benefícios decorrentes destas práticas, como visto em alguns estudos (Gonçalves et al., 2011), puderam ser observados com uma frequência semanal de apenas dois encontros, ao contrário de Telles, Dash e Naveen (2009), que verificaram tais achados com maior frequência (5 encontros). Estes dados evidenciam o potencial de incorporação destas práticas na rotina dos sujeitos, uma vez que sua adoção é capaz de trazer benefícios mesmo com uma reduzida frequência semanal de sessões.
A maior distância alcançada pela modalidade ballness pode ser explicada pelo tipo de trabalho desenvolvido por modalidade. A despeito desta modalidade utilizar o mesmo método para desenvolver a flexibilidade que a ioga, com a utilização da bola suíça, os exercícios em séries e o tempo de execução parecem tornar mais eficientes o desenvolvimento desta capacidade, uma vez que instituem a característica de funcionalidade (Khalsa, 2004). $\mathrm{Na}$ ioga hatha, ao contrário, são utilizadas mais contrações isométricas, utilizando como carga apenas o peso do próprio corpo, geralmente realizada com uma repetição (Khalsa, 2004).

A inclusão do treinamento de força no ballness, pela utilização do peso da bola e do próprio corpo em vários exercícios, aumenta, estimula e produz maior proliferação de tecido conjuntivo em vários grupamentos musculares. Deste modo, os exercícios com bola forçam os limites da amplitude articular e, baseado nessa concepção, o treinamento de força pode auxiliar no ganho de flexibilidade (Thaash; Kell, 1987).

Outro possível fator a levar em consideração é que, durante a prática de ballness ocorre maior ativação dos proprioceptores, órgãos sensoriais aferentes do Sistema Nervoso Central (SNC) presentes nos músculos, ossos e articulações (Pearson, 2000). Esta maior ativação pode ser desencadeada em decorrência do desequilíbrio promovido pela bola em várias situações (Pearson, 2000). Quando se realiza um movimento, consciente ou inconscientemente, esses órgãos ajudam a controlar e ajustar o deslocamento do corpo, como o de recuperar o equilíbrio ao final do movimento (Pearson, 2000), sendo fundamentais para a modulação do movimento humano (Sharma; Gupta; Bijlani, 2008). Assim, o desenvolvimento de qualquer movimento ou sequência de movimentos é registrado de forma contínua, com objetivo de oferecer a base para modificar o comportamento motor, de forma que eles constituem um dos maiores fatores que influenciam a flexibilidade (Bijlani; Vempati; Yadav, 2005).

O presente texto apresenta algumas limitações. A primeira baseia-se no fato de a amostra constituir-se apenas de sujeitos do gênero feminino, impos- 
sibilitando a generalização dos achados a todos os adultos de meia-idade. Outra limitação refere-se à ausência de dados anteriores ao início do estudo, impedindo a comparação das variáveis nos momentos pré e pós-intervenção.

\section{CONCLUSÃO}

Demonstrou-se que a prática regular de ballness e ioga em mulheres de meia-idade, mesmo com baixa frequência semanal, é suficiente para promover melhora da flexibilidade em comparação às sedentárias. Considerando a potencialidade do ballness em melhorar a flexibilidade e que este é o primeiro estudo em que esta modalidade de exercício é investigada, sugere-se a realização de pesquisas adicionais que contemplem outros grupos populacionais (homens, atletas, bem como com jovens e idosos e sujeitos praticantes de outras modalidades), bem como apresentem melhor desenho metodológico (no tocante ao acompanhamento pré, durante e pós-intervenção das variáveis do estudo).

\section{REFERÊNCIAS}

BALASUBRAMANIAN, B.; PANSARE, M. Effect of yoga on aerobic and anaerobic power of muscles. Indian J Physiol Pharmacol, v. 35, n. 4, p. 281-282, 1991.

BAPTISTA, M. R.; DOS SANTOS ALVES, A. Alterações na função respiratória de idosos, induzidas pela prática do yoga. Corpus et Scientia., v. 1, n. 1, p. 1-12, 2005.

BIJLANI, R. L., et al. "A brief but comprehensive lifestyle education program based on yoga reduces risk factors for cardiovascular disease and diabetes mellitus." Journal of Alternative \& Complementary Medicine 11.2 (2005), p. 267-274.

CHEN, K. M. et al. Physical fitness of older adults in senior activity centers after 24-week silver yoga exercises. Journal of clinical nursing, v. 17, n. 19, p. 2.634-2.646, out. 2008.
CHEN, K. et al. "Development and evaluation of a yoga exercise programme for older adults." Journal of advanced nursing 57.4 (2007), p. 432-441.

CLAY, C. C. et al. The metabolic cost of hatha yoga. Journal of strength and conditioning research / National Strength \& Conditioning Association, v. 19, n. 3, p. 604-610, ago. 2005.

DANTAS, E. Alongamento e flexionamento. 5. ed. Rio de Janeiro: Shape, 2005.

DE BARROS, N. F. et al. "Yoga e promoção da saúde". Ciências: Saúde Coletiva [serial on the Internet], p. 1.305-1.014, 2014.

DIBENEDETTO, M. et al. "Effect of a gentle Iyengar yoga program on gait in the elderly: an exploratory study." Archives of Physical Medicine and Rehabilitation 86.9 (2005), p. 1830-1837.

DONNELLY, J. E. et al. Appropriate physical activity intervention strategies for weight loss and prevention of weight regain for adults. Medicine and science in sports and exercise, v. 41, n. 2, p. 459-471, fev. 2009.

GIBNEY, J.; HEALY, M.-L.; SÖNKSEN, P. H. The growth hormone/insulin-like growth factor-I axis in exercise and sport. Endocrine reviews, v. 28, n. 6 , p. 603-624, out. 2007.

GONÇALVES, L. C. et al. Flexibility, functional autonomy and quality of life (QoL) in elderly yoga practitioners. Archives of gerontology and geriatrics, v. 53, n. 2, p. 158-162, 2011.

KHALSA, S. B. S. Yoga as a therapeutic intervention: a bibliometric analysis of published research studies. Indian Journal of Physiology and Pharmacology, v. 48, n. 1, p. 269-285, 2004.

KRINSKI, K. et al. Efeitos cardiovasculares agudos do exercício resistido em idosas hipertensas Acta Scientiarum. Health Science, 30(2), 107-112, 2008.

LOJUDICE, D. C. et al. Balance and gait of elderly people at retirement homes in Catanduva city. Bras Geriatr Gerontol, n. 11, p. 181-189, 2008.

MACY, D. Yoga in America, „Yoga Journal Media Release" 26, February 2008.

MATSUDO, S. M.; MATSUDO, V. K. R.; BARROS NETO, T. L. Atividade física e envelhecimento: aspectos epidemiológicos. Rev Bras Med Esporte, v. 7, n. 1, p. 2-13, 2001. 
MORALES, S. et al. Sedentarism and physical fitness in postmenopausal women. Nutrición hospitalaria, v. 28, n. 4, p. 1.053-1.059, 2013.

OKUMURA, M. K., SILVA, M. C. Ballness: uma nova tendência de prática corporal alternativa. IV Encontro de produção cientifíca e tecnológica, Campo Mourão, PR. 2009.

PEARSON, K. G. Neural adaptation in the generation of rhythmic behavior. Annual Review of Physiology. 2000.

PULLEN, P. R. et al. Benefits of yoga for African American heart failure patients. Medicine and Science in Sports and Exercise, v. 42, n. 4, p. 651657, 2010.

RAFFERTY, A. P. Physical activity patterns among walkers and compliance with public health recommendations. Med Sci Sports Exerc, v. 34, n. 8, p. 1.255-1.261, 2002.

RAUB, J. A. "Psychophysiologic effects of Hatha Yoga on musculoskeletal and cardiopulmonary function: a literature review." The Journal of Alternative \& Complementary Medicine 8.6 (2002): 797-812.

SHARMA, R.; GUPTA, N.; BIJLANI, R. Effect of yoga based lifestyle intervention on subjective well-being. Indian J Physiol Pharmacol, n.52, p. 123-131, 2008.

SHERMAN, K. J. et al. Study protocol Comparison of yoga versus stretching for chronic low back pain: protocol for the Yoga Exercise Self-care (YES) trial. Trialsjournal. Seattle United States. p. 1-17, 2010.

SINGH, M. A. F. "Exercise and aging." Clinics in geriatric medicine 20.2 (2004): 201-221. GAUCHARD, G. C. et al. Beneficial effect of proprioceptive physical activities on balance control in elderly human subjects. Neurosci Lett, v. 273, n. 2, p. 81-84, 1999.

TELLES, S.; DASH, M.; NAVEEN, K. V. Effect of yoga on musculoskeletal discomfort and motor functions in professional computer users. Work (Reading, Mass.), v. 33, n. 3, p. 297-306, jan. 2009.

THAASH, K.; KELL, B. Flexibility and Strength Training. The Journal of Strength \& Conditioning Research, n. 4, p. 74-75, 1987.
WELLS, K. F., and DILLON, E. K. "The sit and reach - a test of back and leg flexibility." Research Quarterly. American Association for Health, Physical Education and Recreation, n. 23, p. 115-118, 1952.

WESTERTERP, K. R. Daily physical activity and ageing." Current Opinion in Clinical Nutrition \& Metabolic Care, n. 3, p. 485-488, 2000.

WILLIAMS, K. A. et al. Effect of Iyengar yoga therapy for chronic low back pain." Pain, n. 115, p. 107-117, 2005.

Recebido em: 28/11/2015

Aceito em: 11/5/2016 